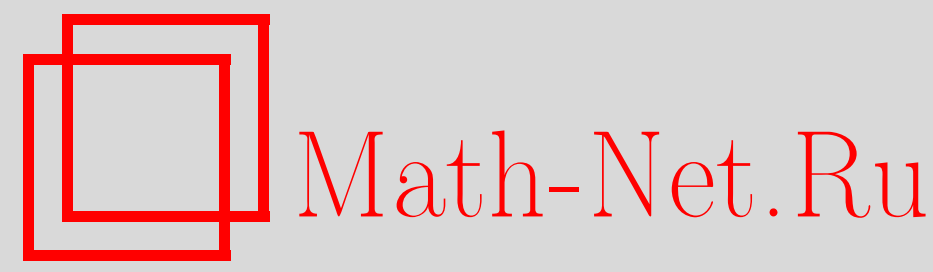

Б. Н. Хабибуллин, Спектральный синтез для пересечения инвариантных подпространств голоморфных функций, Матем. сб., 2005, том 196, номер 3, 119-142

DOI: https://doi.org/10.4213/sm1278

Использование Общероссийского математического портала Math-Net.Ru подразумевает, что вы прочитали и согласны с пользовательским соглашением

http://www.mathnet.ru/rus/agreement

Параметры загрузки:

IP: 54.162 .85 .209

26 апреля 2023 г., $14: 10: 22$ 
УДК $517.982+517.53$

\author{
Б.Н. Хабибуллин
}

\title{
Спектральный синтез для пересечения инвариантных подпространств голоморфных функций
}

\begin{abstract}
Пусть $\Omega$ - вьпуклая область на комплексной плоскости $\mathbb{C} ; H$-пространство голоморфных в $\Omega$ функций с топологией равномерной сходимости на компактах из $\Omega ; W_{1}$ и $W_{2}$ - пара инвариантных (относительно дифференцирования) подпространств в $H$, допускающих спектральный синтез. Даются достаточные условия, при которых пересечение $W_{1} \cap W_{2}$ также допускает спектральный синтез. Следствием этих условий является недавний результат Н. вом конструктивно-количественном обрамлении) о представимости инвариантного подпространства, допускающего спектральньй синтез, в виде пространства решений системы двух однородных уравнений свертки.

Использованы новые аптроксимационные теоремы для целых функций экспоненциального типа.

Библиограф̆ия: 22 названия.
\end{abstract}

\section{Введение}

Всюду в работе под последовательностью чисел (точек) в комплексной плоскости $\mathbb{C}$ понимается пустая, конечная или бесконечная последовательность вида $\Lambda=\left\{\lambda_{n}\right\} \subset \mathbb{C}$, где $n=1,2, \ldots$, не имеющая предельных точек в $\mathbb{C}$. При этом, вообще говоря, в последовательностях допускается повторение точек, т.е. возможно, что $\lambda_{n}=\lambda_{k}$ при $n \neq k$. Для подмножества $B \subset \mathbb{C}$ полагаем $\Lambda(B)=\sum_{\lambda_{n} \in B} 1-$ число точек последовательности $\Lambda$ в $B$. Полагаем $\Lambda(\lambda)=\Lambda(\{\lambda\}), \lambda \in \mathbb{C},-$ дивизор последовательности $\Lambda$. Наряду с последовательностями далее эпизодически встречается совокупность всех точек из $\mathbb{C}$, каждая из которых "считается" счетное число раз. Ее для единообразия тоже относим к последовательностям с дивизором, тождественно равным бесконечности.

Для двух последовательностей $\Lambda$ и $Г$ в $\mathbb{C}$ естественным образом определяются отношения и операции:

1) $\Lambda=\Gamma$, т.е. последовательности $\Lambda$ и говпадают, если $\Lambda(\lambda) \equiv \Gamma(\lambda)$ при всех $\lambda \in \mathbb{C}$, а в противном случае пишем $\Lambda \neq \Gamma$;

2) $\Gamma$ - подпоследовательность последовательности $\Lambda$, если справедливо включение $\Gamma \subset \Lambda$, означаюшее, что $\Gamma(\lambda) \leqslant \Lambda(\lambda)$ при всех $\lambda \in \mathbb{C}$ и при этом определена разность последовательностей $\Lambda \backslash \Gamma$ через разность дивизоров по правилу $(\Lambda \backslash \Gamma)(\lambda) \equiv \Lambda(\lambda)-\Gamma(\lambda), \lambda \in \mathbb{C}$;

Работа вьполнена при поддержке Российского фонда фундаментальных исследований (гранты № № 03-01-00033, 02-01-00030) и Программы поддержки ведущих научных шшкол РФ (грант № НШ-1528.2003.1).

(C) Б. Н. ХАвивуллин 2005 
3) обвединение $\Lambda \cup \Gamma$ и пересечение $\Lambda \cap \Gamma$ последовательностей $\Lambda$ и Г определяются соответственно тождествами $(\Lambda \cup \Gamma)(\lambda) \equiv \Lambda(\lambda)+\Gamma(\lambda)$ и $(\Lambda \cap \Gamma)(\lambda) \equiv$ $\min \{\Lambda(\lambda), \Gamma(\lambda)\}, \lambda \in \mathbb{C}$

4) симметрическую разность $\Lambda \Delta \Gamma$ последовательностей $\Lambda$ и Г определяет равенство $\Lambda \Delta \Gamma=\Lambda \cup \Gamma-\Lambda \cap \Gamma$ или эквивалентное ему тождество $(\Lambda \Delta \Gamma)(\lambda) \equiv \max \{\Lambda(\lambda), \Gamma(\lambda)\}, \lambda \in \mathbb{C}$.

Легко видеть, что введенные отношения и операции над последовательностями обладают свойствами, совпадающими со свойствами аналогичных теоретико-множественных отношений и операций.

Каждой последовательности $\Lambda$ в $\mathbb{C}$ сопоставляем систему (кратных) экспонент

$$
\operatorname{Exp}_{\Lambda}=\left\{z^{k-1} e^{\lambda z}: \lambda \in \Lambda, 1 \leqslant k \leqslant \Lambda(\lambda), k=1,2, \ldots\right\}, \quad z \in \mathbb{C}
$$

При этом $\operatorname{Exp}_{\Lambda} \cap \operatorname{Exp}_{\Gamma}=\operatorname{Exp}_{\Lambda \cap \Gamma}$, но $\operatorname{Exp}_{\Lambda} \cup \operatorname{Exp}_{\Gamma}=\operatorname{Exp}_{\Lambda \Delta \Gamma}$ (слева - обычные теоретико-множественные пересечение и объединение).

Всюду далее $\Omega$ - область ${ }^{1}$ в $\mathbb{C}$. Через $H(\Omega)$ обозначаем локально выпуклое пространство голоморфных в $\Omega$ функций над $\mathbb{C}$, наделенное топологией равномерной сходимости на компактах. Там, где это не вызывает разночтений, пространство $H(\Omega)$ обозначаем одним символом $H$.

Напомним некоторые понятия и сведения по спектральному синтезу на области в $\mathbb{C}$ из основополагающих для настоящей статьи работ И.Ф. Красичкова-Терновского [1], [2], представляющих собой определяющий задел обширной программы его исследований по этой теме и содержащих основные исторические сведения об истоках проблематики.

Замкнутое линейное подпространство $W$ в $H$ называем инвариантным (относительно дифференцирования), если вместе с каждой функцией $f \in W$ оно содержит и ее производную $f^{\prime} \in W$. Очевидно, множество всех инвариантных подпространств на $\Omega$ замкнуто относительно пересечения любого числа таких подпространств. Спектром нетривиального $(\neq\{0\}, H)$ инвариантного подпространства $W$ называем максимальную относительно включения последовательность $\Lambda$, для которой $\operatorname{Exp}_{\Lambda} \subset W$. Спектр инвариантного подпространства обозначаем далее через $\operatorname{Spec} W$. Спектры тривиальных инвариантных подпространств $\{0\}$ и $H-$ соответственно пустое множество и множество повторяющихся счетное число раз точек плоскости $\mathbb{C}$, т.е. в терминах дивизоров $(\operatorname{Spec}\{0\})(\lambda) \equiv 0$ и $(\operatorname{Spec} H)(\lambda) \equiv \infty$, $\lambda \in \mathbb{C}$.

Инвариантное подпространство $W \subset H$ допускает спектральный синтез (на $\Omega$ ), если замыкание $\overline{\left\langle\operatorname{Exp}_{\mathrm{Spec} W}\right\rangle}$ в пространстве $H$ линейной оболочки $\left\langle\operatorname{Exp}_{\text {Spec } W}\right\rangle$ системы $\operatorname{Exp}_{\text {Spec } W}$ совпадает с пространством $W$, или, иначе говоря, система $\operatorname{Exp}_{\text {Spec } W}$ полна в $W$. Таким образом, спектр инвариантного подпространства, допускающего спектральный синтез, однозначно определяет само подпространство. Возможна и другая характеризация: инвариантное подпространство $W$ допускает спектральный синтез тогда и только тогда, когда оно минимальное (относительно отношения включения) среди всех инвариантных подпространств со спектром $\mathrm{Spec} W$.

\footnotetext{
${ }^{1}$ Области и компакты в $\mathbb{C}$, если не оговорено противное, непустые.
} 
Задача спектрального синтеза для инвариантного подпространства $W \subset H$ - найти условия, при которых $W$ допускает спектральный синтез.

Наиболее содержательные и полные результаты по спектральному синтезу в $H$ получены в случае, когда область $\Omega$ выпукла. И. $Ф$. Красичков-Терновский доказал [1; теорема 6.1], что пространство решений однородного уравнения свертки в выпукклой области $\Omega$ (обязательно инвариантное) всегда допускает спектральныцй синтез (см. ниже). Как показали И. Ф. Красичков-Терновский $[2 ;$ теорема 8.1] и несколько позже другим методом и в менее обшей форме А. Ф. Леонтьев [3], [4; теорема 5.2.8], всякое инвариантное подпространство на неограниченной выпуклой области $\Omega$ допускает спектральный синтез. В случае невыпуклой области $\Omega$ задача спектрального синтеза в $H$ исследовалась, например, И. Ф. Красичковым-Терновским [5] в рамках задачи распространения спектрального синтеза и имеет свою специфику. В связи с этим далее всюду задача спектрального синтеза рассматривается только на выпуклой ограниченной области.

Основная исследуемая нами проблема в несколько менее конкретной форме была сформулирована И.. К. Красичковым-Терновским в $[2 ; \S 6]$ :

ЗАДАЧА О ПЕРЕСЕЧЕНИИ ИНВАРИАНТНЫХ ПОДПРОСТРАНСТВ. При условии, что инвариантные подпространства $W_{1}, W_{2}, \ldots, W_{n}$ на области $\Omega$ допускают спектральный синтез, исключительно в терминах спектров $\mathrm{Spec} W_{1}$, Spec $W_{2}, \ldots$, Sрес $W_{n}$ и области $\Omega$ выяснить, допускает ли спектральный синтез пересечение $W=\bigcap_{k=1}^{n} W_{k}$.

Мотивировкой такой постановки служит прежде всего задача аппроксимации решений системы однородных уравнений свертки линейными комбинациями элементарных решений.

Конкретнее, пусть $\mathscr{S} \in H^{*}(\Omega)$. Здесь и далее через $H^{*}(\Omega)$ или $H^{*}$ обозначается пространство линейных непрерывных функционалов на $H ;\langle\mathscr{S}, f\rangle$ - действие функционала $\mathscr{S}$ на функцию $f$. По теореме $\Phi$. Рисса функционал $\mathscr{S}$ может быть представлен в интегральной форме $\langle\mathscr{S}, f\rangle=\int f d \sigma, f \in H$, где $\sigma$ - некоторая борелевская комплекснозначная мера с компактным носителем supp $\sigma \subset \Omega$. Следовательно, определена свертка “*” этого функционала с функциями $f \in H$, действуюшая из $H$ в $H\left(\omega_{\mathscr{S}}\right)$, где $\omega_{\mathscr{S}}=\{h \in \mathbb{C}: \operatorname{supp} \sigma+h \subset \Omega\}$ - геометрическая разность области $\Omega$ и компакта $\operatorname{supp} \sigma$ :

$$
(\mathscr{S} * f)(h)=\langle\mathscr{S}, f(\zeta+h)\rangle=\int_{\operatorname{supp} \sigma} f(\zeta+h) d \sigma(\zeta), \quad f \in H, \quad h \in \omega_{\mathscr{S}}
$$

Таким образом, имеет смысл однородное уравнение свертки

$$
\left((\mathscr{S} * f)(h) \equiv 0, h \in \omega_{\mathscr{S}}\right) \Longleftrightarrow(\mathscr{S} * f=0) .
$$

Пространство его решений образует инвариантное подпространство $W_{\mathscr{S}}$ на $\Omega$. Функционалу $\mathscr{S} \in H^{*}$ преобразование Лапласа

$$
T: \mathscr{S} \rightarrow S(z)=\left\langle\mathscr{S}, e^{z \zeta}\right\rangle, \quad z \in \mathbb{C}
$$


сопоставляет характеристическую функиию $S$, которая является целой функцией экспоненциального типа с конечньм индикатором роста

$$
h_{S}(\theta)=\limsup _{r \rightarrow+\infty} r^{-1} \ln \left|S\left(r e^{i \theta}\right)\right|, \quad \theta \in \mathbb{R} .
$$

Последовательность Zero $S \subset \mathbb{C}$ всех нулей характеристической функции $S$, перенумерованная с учетом кратности (каждая точка встречается в последовательности Zero $_{S}$ ровно столько раз, какова кратность нуля функции $S$ в этой точке), и есть спектр инвариантного подпространства $W_{\mathscr{S}}$, который далее называем также спектром уравнения (0.2). При этом система $\operatorname{Exp}_{Z_{\text {Zero }}}$ - это әлементарные решения уравнения (0.2). Как уже отмечалось выше, всякое такое инвариантное подпространство $W \mathscr{S}$ допускает спектральньй синтез. Положение существенно усложняется при рассмотрении системы $n>1$ однородных уравнений свертки

$$
\left\{\begin{array}{l}
\mathscr{S}_{1} * f=0 \\
\mathscr{S}_{2} * f=0 \\
\ldots \ldots \ldots \\
\mathscr{S}_{n} * f=0
\end{array}\right.
$$

Пусть $S_{k}=T\left(\mathscr{S}_{k}\right)$ - характеристическая функция функционала $\mathscr{S}_{k}$. В то время как пространства решений, соответственно $W_{1}, W_{2}, \ldots, W_{n}$, каждого уравнения в отдельности допускают спектральный синтез, для инвариантного подпространства, являюшегося пространством решений $\bigcap_{k=1}^{n} W_{k}$ системы $(0.4)$, со спектром $\bigcap_{k=1}^{n} \operatorname{Spec} W_{k}=\bigcap_{k=1}^{n}$ Zero $_{S_{k}}$ ситуация приобретает альтернативный характер [2]. Она полностью определяется взаимным расположением точек из последовательностей $\operatorname{Spec} W_{k}=\operatorname{Zero}_{S_{k}}, k=1,2, \ldots, n$, и связью его с геометрией области $\Omega$. Таким образом, вопрос о возможности аппроксимации каждого решения системы (0.4) линейными комбинациями элементарных решений - это частньй случай задачи о пересечении инвариантных подпространств, и даже он на настояшее время еше очень далек от окончательного решения.

В работе рассматривается задача о пересечении только двух инвариантных подпространств. И в этом случае известно не очень много (см. $\S 1$ ), а законченное решение этой задачи пока не просматривается. В то же время каждое достаточное условие допустимости спектрального синтеза для пересечения двух инвариантных подпространств поставляет целый набор таковых для бо́льшего числа подпространств в зависимости от того, в какой последовательности путем пересечения пар подпространств “добираться до конечного пункта" $\bigcap_{k=1}^{n} W_{k}$. Кроме того, недавно в работе Н.Ф. Абузлровой [6; теорема 1] (см. также [7; теорема 4.6]) установлено, что каждое инвариантное подпространство $W$ в $H$, допускающее спектральный синтез, может быть представлено как пространство решений системы двух однородных уравнений свертки, т.е. как пересечение двух специальных инвариантных подпространств, допускающих спектральный синтез. Последнее дополнительно стимулирует исследование задачи о пересечении именно двух инвариантных подпространств. В $\S 1$ результат Н. $\Phi$. Абузяровой приводится в несколько более детализированной конструктивно-количественной форме как следствие основной теоремы, сформулированной там же и доказанной в $\S 5$ (см. также замечание в конце $\S 5$ ) . 
Ряд общих условий на пространства голоморфных и иных функций, при которых каждое инвариантное подпространство, допускающее спектральньй синтез, представляется в виде пространства решений не более чем двух уравнений типа свертки, можно извлечь из двойственной трактовки общих результатов автора [8]-[10] о возможности локального описания и о 2-порожденности замкнутых подмодулей над кольцом многочленов $\mathbb{C}[z]$ в пространствах голоморфных функций. В количественном отношении эти общие условия в значительной мере не “дотягивают" до основной теоремы настояшей работы.

Основная теорема показывает, что при определенной “близости” спектров двух инвариантных подпространств, допускающих спектральный синтез, их пересечение также допускает спектральный синтез. Как и в [1], [2], [5], [6], доказательство основной теоремы и несколько более сильной теоремы 3 из $\S 5$ опирается прежде всего на обшую двойственную схему И. $\Phi$. Красичкова-Терновского из [1], [2], сводяшую задачу спектрального синтеза к задаче локального описания замкнутых подмодулей над кольцом $\mathbb{C}[z]$ в пространстве целых функций экспоненциального типа с ограничениями на их индикаторы роста. Применение этой схемы осушествляется с помошью аппроксимационной теоремы 2 из 44 об аппроксимации тождественного нуля специальной последовательностью целых функций экспоненциального типа. При этом аппроксимационные теоремы 1 и 2 наряду с тем, что во многом продиктованы именно этой схемой, представляют, по-видимому, и самостоятельный интерес.

Изложение замкнуто в себе в том смысле, что формулировки определений приводятся в полном объеме, а аппроксимационная теорема из [11] включена в формулировку уточняюшей ее теоремы 1 из $\S 2$.

В $\S 3$ даны оценки сверху многочленов, которые применяются при доказательстве теоремы 2 , но могут быть полезны и в других вопросах. В последнем $\S 6$ построен пример, устанавливаюший "верхнюю границу близости" спектров двух допускаюших спектральный синтез инвариантных подпространств, начиная с которой уже нельзя гарантировать наследования этого свойства их пересечением. Зазор между этой верхней границей близости спектров и близостью спектров в основной теореме пока весьма значителен. В связи с этим в конце $\S 6$ выдвигается гипотеза, подтверждение которой означало бы законченное в первом приближении решение задачи о пересечении двух инвариантных подпространств в терминах близости их спектров.

Автор признателен Н. Ф. Абузяровой за предоставленные полезные материалы, а также рецензенту за ценные замечания.

Настоящая работа посвящается семидесятилетию моего учителя И. Ф. Красичкова-Терновского.

\section{$\S 1$. Формулировки основного и предшествовавших ему результатов; сравнительный анализ}

Для подмножеств $A, B \subset \mathbb{C}$ потребуются следующие обозначения: $\bar{A}$ и $\partial A-$ соответственно замыкание и граница множества $A$; запись $A \Subset B$ означает, что $\bar{A}$ - относительно компактное подмножество в $B$ в индуцированной евклидовой топологии; $\operatorname{dist}(A, B)-$ евклидово расстояние между $A$ и $B ; B^{*}=\{\bar{z}: z \in B\}$ - множество, симметричное $B$ относительно вещественной оси; $p(B)$ - 
периметр $B$, т.е. длина границы $\partial B$ при условии, что она спрямляема;

$$
K_{B}(\theta)=\sup \left\{\operatorname{Re} z e^{-i \theta}: z \in B\right\}, \quad \theta \in \mathbb{R}
$$

- опорная функция множества $B$.

Далее по теории целых функций в основном используются факты и терминология из книг Б. Я. Левина [12], [13], а по спектральному синтезу - из работ И. Ф. Красичкова-Терновского [1], [2].

Пусть $f$ - целая функция, не равная тождественно нулю (обозначаем $f \not \equiv 0$ ). Последовательность $\Lambda \subset \mathbb{C}$ назьваем последовательностью нулей функиии $f \in$ $H(\mathbb{C})$, если кратность нуля функции $f$ в каждой точке $\lambda \in \mathbb{C}$ равна числу повторений точки $\lambda$ в последовательности $\Lambda$ (пишем Zero $f=\Lambda$ ). Говорим, что функция $f \in H(\mathbb{C})$ обращается в нуль на последовательности $\Lambda \subset \mathbb{C}$, если кратность нуля функции $f$ в каждой точке $\lambda \in \mathbb{C}$ не меньше числа повторений точки $\lambda$ в последовательности $\Lambda$ (пишем $f(\Lambda)=0$ ).

Пусть $E$ - некоторое линейное пространство целых функций. Последовательность $\Lambda \subset \mathbb{C}$ называем последовательностью неединственности для пространства $E$, если существует функция $f \in E$ такая, что $f \not \equiv 0$ и $f(\Lambda)=0$.

Пусть $\Omega$ - выпуклая область в $\mathbb{C}$ с опорной функцией $K_{\Omega}$. Через $P_{\Omega}$ обозначаем пространство целых функций экспоненциального типа $L$ с индикатором роста $h_{L}(\theta)<K_{\Omega}(\theta), \theta \in \mathbb{R}$. Преобразование Лапласа (0.3) задает линейный изоморфизм пространства $H^{*}$ на пространство $P_{\Omega^{*}}$, которое далее часто обозначаем одним символом $P$. Последовательность неединственности $\Lambda \subset \mathbb{C}$ для $P$ будем называть также последовательностью неединственности для выпуклой облас$m u \Omega$. В силу известной взаимосвязи меж ду полнотой и единственностью система $\operatorname{Exp}_{\Lambda}$ не полна в $H$ тогда и только тогда, когда $\Lambda$ - последовательность неединственности для $\Omega$.

Каждой последовательности $\Lambda$ в $\mathbb{C}$ сопоставляем в $H$ инвариантное подпространство $W(\Lambda) \stackrel{\text { def }}{=} \overline{\left\langle\operatorname{Exp}_{\Lambda}\right\rangle}$. Очевидно, подпространствами такого вида исчерпываются все инвариантные подпространства, допускающие спектральный синтез. Для нетривиального подпространства $W(\Lambda)$ его спектр совпадает с последовательностью $\Lambda$.

$\mathrm{K}$ первым содержательньм результатам по задаче о пересечении инвариантных подпространств можно отнести доказанную И. $\Phi$. Красичковым-Терновским $[2 ;$ предложение 6.5$]$ и приведенную ниже теорему А, которая формулируется здесь в терминах спектров.

Теорема А (И.Ф. Красичков-Терновский). Если для последовательностей $\Lambda_{1}, \Lambda_{2}, \ldots, \Lambda_{n}$ их симметрическая разность $\Lambda_{1} \Delta \Lambda_{2} \Delta \cdots \Delta \Lambda_{n}-$ последовательность неединственности для выпуклой области $\Omega$, то пересечение $\bigcap_{k=1}^{n} W\left(\Lambda_{k}\right)$ допускает спектральный синтез.

В работе [2; предложение 6.5] установлено, что если замыкание $\overline{\sum_{k=1}^{n} W\left(\Lambda_{k}\right)}$ суммы $\sum_{k=1}^{n} W\left(\Lambda_{k}\right)$ инвариантных подпространств $W\left(\Lambda_{k}\right)$ - нетривиальное подпространство, т.е. не совпадает с $H$, то их пересечение $\bigcap_{k=1}^{n} W\left(\Lambda_{k}\right)$ допускает 
спектральный синтез. Эта исходная формулировка эквивалентна теореме А. Действительно, по теореме Хана-Банаха нетривиальность подпространства

$$
\overline{\sum_{k=1}^{n} W\left(\Lambda_{k}\right)} \neq H
$$

эквивалентна существованию ненулевого функционала $\mathscr{S} \in H^{*}$, аннулирующего это подпространство или аннулируюшего каждую из систем $\operatorname{Exp}_{\Lambda_{k}}, k=1,2, \ldots$. Другими словами, это равносильно аннулированию системы

$$
\bigcup_{k=1}^{n} \operatorname{Exp}_{\Lambda_{k}}=\operatorname{Exp}_{\Lambda_{1} \Delta \Lambda_{2} \Delta \cdots \Delta \Lambda_{n}}
$$

некоторьм функционалом $\mathscr{S} \neq 0$ из $H^{*}$. Для характеристической функции $S \in P$ из (0.3) функционала $\mathscr{S}$ последнее в точности означает, что $S \not \equiv 0$ и

$$
S\left(\Lambda_{1} \Delta \Lambda_{2} \Delta \cdots \Delta \Lambda_{n}\right)=0,
$$

т.е. $\Lambda_{1} \Delta \Lambda_{2} \Delta \cdots \Delta \Lambda_{n}-$ последовательность неединственности для $\Omega$.

Теорема $\mathrm{A}$, как и результаты настоящей статьи, наряду с другими вопросами теории функций (интерполяция, аналитическое продолжение и пр.) подчеркивает острую актуальность задачи законченного описания последовательностей неединственности для ограниченной выпуклой области $\Omega$ или эквивалентной ей проблемы описания всех полных систем экспонент $\operatorname{Exp}_{\Lambda}$ в пространстве $H$ исключительно в терминах определенного мажорирования различных плотностей последовательности показателей $\Lambda$ соответствующими геометрическими характеристиками области $\Omega$. В то время как законченное решение этой задачи для неограниченной выпуклой области $\Omega$ в форме критерия полноты системы экспонент было получено в работе автора [14; теорема 2], удовлетворительного описания последовательностей неединственности, к сожалению, до сих пор не сушествует ни для какой ограниченной выпуклой области $\Omega$ (подробнее по этому поводу см. [15] и наш краткий обзор [16]). Здесь на этой задаче мы не останавливаемся, поскольку она далеко не тривиальна и требует отдельного рассмотрения.

Другой аспект, обеспечивающий допустимость спектрального синтеза для пересечений пространств решений двух уравнений свертки, - определенная "близость" спектров этих уравнений - по-видимому, впервые нашел отражение в фактически ключевом для ее работы результате Н.. . Абузяровой [6; предложение] (см. также [7; теорема 3.1], [17; теорема 1]). Он сформулирован в оригинале как достаточное условие возможности локального описания замкнутого подмодуля над кольцом $\mathbb{C}[z]$, порожденного двумя функциями, в топологическом модуле $P$ над $\mathbb{C}[z]$. Здесь для удобства сравнения с основным результатом статьи приведем его в двойственной (см. [1], [2]) формев виде условия допустимости спектрального синтеза для пересечения двух инвариантных подпространств.

Teорема В (Н.Ф. Абузярова). Пусть $\Lambda=\left\{\lambda_{n}\right\} u \Lambda^{\prime}, \quad \Gamma=\left\{\gamma_{n}\right\} u \Gamma^{\prime}-$ четыре последовательности в $\mathbb{C}$, являющиеся последовательностями нулей цельх функиий әкспоненциального типа соответственно $F u f, G u g$, $0 \notin \Lambda \cup \Gamma ; \quad \Omega$ - ограниченная выпуклая область в $\mathbb{C} ;$ разложения Адамара 
для $F$ и $G$ имеют вид

$$
F(z)=e^{a z} \prod_{n=1}^{\infty}\left(1-\frac{z}{\lambda_{n}}\right) \exp \frac{z}{\lambda_{n}}, \quad G(z)=e^{b z} \prod_{n=1}^{\infty}\left(1-\frac{z}{\gamma_{n}}\right) \exp \frac{z}{\gamma_{n}}
$$

Предположим, что функиии $\mathscr{F}=f F$ и $\mathscr{G}=g G$ принадлежсат $P$.

Положим

$$
\delta_{0}=\left|a-b+\sum_{n=1}^{\infty}\left(\frac{1}{\lambda_{n}}-\frac{1}{\gamma_{n}}\right)\right|, \quad \Delta=\max \left\{\sup _{n} \frac{n}{\left|\lambda_{n}\right|}, \sup _{n} \frac{n}{\left|\gamma_{n}\right|}\right\}
$$

$\mu(\chi)$ - функция, обратная к функиии $\chi(\mu)=(1 / \mu) \ln (1+\mu)+\ln (1+1 / \mu)$. Предположим, что существуют число $\delta>0$ и такая тригонометрически выпуклая функиия $h(\theta)$, что $h(\theta)+\delta_{0}+\delta<K_{\Omega^{*}}(\theta), \theta \in \mathbb{R}$. Если существует постоянная $C$ такая, что для всех $z=r e^{i \theta} \in \mathbb{C}, r \geqslant 0$, выполнень оценки $|f(z) \mathscr{G}(z)| \leqslant C \exp (h(\theta) r), \quad|g(z) \mathscr{F}(z)| \leqslant C \exp (h(\theta) r)$ и справедливо соотношение

$$
\liminf _{n \rightarrow \infty} \frac{\ln \left(\sum_{k \geqslant n}\left|1 / \lambda_{k}-1 / \gamma_{k}\right|\right)}{\max _{k \leqslant n}\left\{\left|\lambda_{k}\right|,\left|\gamma_{k}\right|\right\}}<-\mu\left(\frac{\delta}{\Delta}\right)\left(\frac{h}{\sqrt{3}}+\delta\right)
$$

где $h=\max _{\theta \in \mathbb{R}}(h(\theta+\pi / 2)-h(\theta-\pi / 2))$, то инвариантное подпространство $W\left(\Lambda \cup \Lambda^{\prime}\right) \cap W\left(\Gamma \cup \Gamma^{\prime}\right)$ допускает спектральный синтез в $H$.

Приведем основной результат настоящей статьи, который следует из несколько более сильной теоремы 3 из $\S 5$. Полагаем $\ln ^{+} t=\max \{0, \ln t\}$.

ОСНовная ТеОРема. Пусть $\Lambda=\left\{\lambda_{n}\right\} u \Lambda^{\prime}, \quad \Gamma=\left\{\gamma_{n}\right\} u \Gamma^{\prime}$ - четыр следовательности в $\mathbb{C}$ и $\Lambda \cup\left(\Lambda^{\prime} \triangle \Gamma^{\prime}\right)$ - последовательность неединственности для некоторой выпуклой области $D \Subset \Omega$. Положсим $d=\operatorname{dist}(D, \partial \Omega)$, $p=p(D)-$ периметр D. Eсли

$$
\liminf _{n \rightarrow \infty} \frac{\ln \left(\sum_{k \geqslant n}\left|1 / \lambda_{k}-1 / \gamma_{k}\right|\right)}{\max _{k \leqslant n}\left|\lambda_{k}\right|} \leqslant-\frac{p}{2 \pi}\left(1+e \ln +\frac{e p}{2 \pi d}\right)
$$

то $W\left(\Lambda \cup \Lambda^{\prime}\right) \cap W\left(\Gamma \cup \Gamma^{\prime}\right)$ допускает спектральный синтез в $H$.

Отметим, что в основной теореме, если исключить тривиальную постановку, последовательность $\Lambda \cup \Lambda^{\prime}$ обязана быть последовательностью неединственности для некоторой выпуклой области $D \Subset \Omega$.

Теорема А для $n=2$ получается из основной теоремы, если положить в ней $\Lambda=\Gamma=\varnothing$, а последовательности $\Lambda^{\prime}, \Gamma^{\prime}$ переобозначить соответственно как $\Lambda_{1}, \Lambda_{2}$. Относительно теоремы В заметим, что, во-первых, переход от последовательностей нулей целых функций экспоненциального типа в теореме В к последовательности неединственности в основной теореме, равно как и замена априорных условий теоремы В на несколько целых функций одним условием лишш на последовательность $\Lambda \cup\left(\Lambda^{\prime} \Delta \Gamma^{\prime}\right)$, уже значительно расширяет сферу применимости основной теоремы по сравнению с теоремой В. Во-вторых, правая часть неравенства (1.3) теоремы В существенно зависит даже от малых “шевелений” отдельных 
точек последовательностей $\Lambda$ и Г. Например, правая часть в (1.3) может значительно уменшшиться при смешении в малую окрестность нуля хотя бы одной точки из $\Lambda$ или $\Gamma$ (см. определение величины $\Delta$ в (1.2) и ее участие в условии (1.3); при $\chi \rightarrow 0$ порядок роста функции $\mu(\chi)$ близок к $1 / \chi)$. Это означает, что в отличие от условия (1.4) основной теоремы условие (1.3) в теореме В не асимптотическое относительно распределения точек из $\Lambda$ или $\Gamma$, хотя по сути рассматриваемой задачи обязано быть таковым. Ради справедливости отметим, что эту погрешность в определенной степени можно ликвидировать и в рамках доказательства Н. $\Phi$. Абузяровой теоремы В.

СлЕДСТВИЕ. Пусть нетривиальное инвариантное подпространство $W$ из $Н$ допускает спектральный синтез, а последовательность почек $\Lambda=\left\{\lambda_{n}\right\}$ дополняет спектр $\operatorname{Spec} W$ до последовательности нулей $\operatorname{Zero}_{L}=\Lambda \cup \operatorname{Spec} W$ характеристической функиии $L \not \equiv 0$ некоторого функционала $\mathscr{L} \in H^{*}(D) \subset$ $H^{*}$, әде $D \Subset \Omega-$ выпуклая область, т.е. $h_{L}<K_{D^{*}}$. Тогда для любой последовательности $\Gamma=\left\{\gamma_{n}\right\}$, не пересекающейся $c \Lambda$ и удовлетворяющей в обозначениях основной теоремы условию (1.4), найдется такой функционал $\mathscr{G} \in H^{*}(D)$, что для его характеристической функиии $G$ имеют место равенства $\operatorname{Zero}_{G}=\Gamma \cup \operatorname{Spec} W$ и $h_{G}=h_{L}$, а подпространство $W$ при этом совпадает с пространством решений системь

$$
\left\{\begin{array}{l}
\mathscr{L} * f=0 \\
\mathscr{G} * f=0
\end{array}\right.
$$

Следует отметить, что для любого нетривиального инвариантного подпространства в $H$ существует бесконечное разнообразие способов дополнения его спектра до последовательности нулей характеристической функции ненулевого функционала из $H^{*}(D)$, если выпуклая область $D \Subset \Omega$ достаточно велика. Таким образом, приведенное следствие содержательно для любого $W$, допускающего спектральный синтез. Если отвлечься от его конструктивно-количественного обрамления, в качественном смысле оно совпадает с результатом Н. Ф. Абузяровой [6; теорема 1] о возможности представления такого $W$ в виде пространства решений некоторой системы (1.5).

\section{§ 2. Первая аппроксимационная теорема}

Пусть $L$ - целая функция. При условии $L(\lambda)=0, \gamma \in \mathbb{C}$, как и в работе [11], введем следующее обозначение:

$$
L_{[\lambda]}^{[\gamma]}(z)=\frac{1-z / \gamma}{1-z / \lambda} L(z), \quad \lambda \cdot \gamma \neq 0 .
$$

ПРЕДЛОЖЕНИЕ 2.1. Пусть $L-$ целая функция, $L(\lambda)=0, \lambda \neq 0$. Тогда для комплексной точки $\gamma \neq 0$ для любого числа $\delta>0$ выполнена оченка

$$
\left|L(z)-L_{[\lambda]}^{[\gamma]}(z)\right| \leqslant \frac{|z|}{\delta}\left|\frac{1}{\lambda}-\frac{1}{\gamma}\right| \cdot \begin{cases}|L(z)|, & |z-\lambda| \geqslant \delta|\lambda|, \\ \max _{|\zeta-\lambda|=\delta|\lambda|}|L(\zeta)|, & |z-\lambda|<\delta|\lambda| .\end{cases}
$$


ДокАЗАТЕЛЬСТво. Воспользуемся тождеством

$$
L(z)-L_{[\lambda]}^{[\gamma]}(z) \equiv L(z) \frac{z \lambda}{z-\lambda}\left(\frac{1}{\lambda}-\frac{1}{\gamma}\right), \quad z \in \mathbb{C} .
$$

Отсюда при $|z-\lambda| \geqslant \delta|\lambda|$ имеем

$$
\left|L(z)-L_{[\lambda]}^{[\gamma]}(z)\right| \leqslant|L(z)| \frac{|z \lambda|}{|z-\lambda|}\left|\frac{1}{\lambda}-\frac{1}{\gamma}\right| \leqslant \frac{|z||\lambda|}{\delta|\lambda|}\left|\frac{1}{\lambda}-\frac{1}{\gamma}\right||L(z)|,
$$

что дает первую строку в (2.2).

При $|z-\lambda|<\delta|\lambda|$ из $(2.3)$ по принципу максимума для целой функции $L(z) /(z-\lambda)$ получаем

$$
\left|L(z)-L_{[\lambda]}^{[\gamma]}(z)\right| \leqslant \max _{|\zeta-\lambda|=\delta|\lambda|}\left|\frac{L(\zeta)}{\zeta-\lambda}\right||z \lambda|\left|\frac{1}{\lambda}-\frac{1}{\gamma}\right|=\frac{|z||\lambda|}{\delta|\lambda|}\left|\frac{1}{\lambda}-\frac{1}{\gamma}\right| \cdot \max _{|\zeta-\lambda|=\delta|\lambda|}|L(\zeta)|,
$$

что завершает доказательство (2.2).

Воспроизведем одно определение из [11; определение 1].

Пусть $L \not \equiv 0$ - целая функция и $\Lambda=\left\{\lambda_{n}\right\} \subset$ Zero $_{L}$, а $\Gamma=\left\{\gamma_{n}\right\}$ - последовательность в $\mathbb{C}, n=1,2, \ldots$, и $0 \notin \Lambda \cup \Gamma$.

Рекуррентно заданную последовательность целых функций

$$
L_{1}(z)=L(z), \quad L_{n+1}(z)=\left(L_{n}\right)_{\left[\lambda_{n}\right]}^{\left[\gamma_{n}\right]}(z), \quad n=1,2, \ldots,
$$

где функции вида $L_{[\lambda]}^{[\gamma]}$ определены в $(2.1)$, называем жанонической последовательностью (рода 0) для функиии L, сдвигающей подпоследовательность нулей $\Lambda \subset \operatorname{Zero}_{L}$ в последовательность $Г$.

Tеорема 1 (аппроксимационная). Пусть $L \not \equiv 0$ - целая функция әкспоненииального типа с индикатором роста $h_{L}, \Lambda=\left\{\lambda_{n}\right\} \subset$ Zero $_{L}-$ nодпоследовательность нулей функиии $L, a \Gamma=\left\{\gamma_{n}\right\}-$ последовательность в $\mathbb{C}$, $n=1,2, \ldots, \quad 0 \notin \Lambda \cup \Gamma$, и пусть сходится ряд

$$
\sum_{k=1}^{\infty}\left|\frac{1}{\lambda_{k}}-\frac{1}{\gamma_{k}}\right|<\infty
$$

Тогда каноническая последовательность цельх функиий әкспоненциального типа $\left\{L_{n}\right\}$, сдвигающая подпоследовательность нулей $\Lambda$ в последовательность $\Gamma$, сходится равномерно на компактах из $\mathbb{C} \kappa$ целой функции әкспоненциального типа $G \not \equiv 0, G(0)=L(0)$, с индикатором роста $h_{G}=h_{L} u$ с последовательностью нулей $\operatorname{Zero}_{G}=\left(\operatorname{Zero}_{L} \backslash \Lambda\right) \cup \Gamma$. При этом:

а) для любого числа $\varepsilon>0$ найдется постоянная $A_{\varepsilon}$ такая, что последовательность $\left\{L_{n}\right\}$ удовлетворяет равномерной по п оченке

$\left|L_{n}(z)\right| \leqslant A_{\varepsilon} \exp \left(\left(h_{L}(\theta)+\frac{\varepsilon}{3}\right) r\right), \quad z=r e^{i \theta} \in \mathbb{C}, \quad r \geqslant 0, \quad n \in \mathbb{N} ;$

b) скорость сходимости такова, что для любого числа $\varepsilon>0$ найдется постоянная $C_{\varepsilon}$, с которой при всех $z=r e^{i \theta} \in \mathbb{C}, r \geqslant 0$, и при всех $n=1,2, \ldots$ выполнень оценки

$$
\left|L_{n}(z)-G(z)\right| \leqslant C_{\varepsilon} \sum_{k=n}^{\infty}\left|\frac{1}{\lambda_{k}}-\frac{1}{\gamma_{k}}\right| \cdot \exp \left(\left(h_{L}(\theta)+\varepsilon\right) r\right) .
$$


ДокАЗАТЕЛЬСтво. Все утверждения теоремы, включая п. а), за исключением утверждения о скорости сходимости из п. b), доказаны в [11; аппроксимационная теорема 1]. Докажем п. b).

В силу равномерной непрерывности тригонометрически вьпуклой функции $h_{L}$ можно подобрать число $\delta>0$ столь малым, что выполнена оценка

$$
\max \left\{\left|\left(h_{L}(\theta)+\frac{\varepsilon}{3}\right) r-\left(h_{L}(\varphi)+\frac{\varepsilon}{3}\right) t\right|:\left|r e^{i \theta}-t e^{i \varphi}\right| \leqslant 2 \delta t, r, t \geqslant 0\right\} \leqslant \frac{\varepsilon}{3} r .
$$

Это вместе с (2.6) и оценкой (2.2) предложения 2.1 дает оценку

$$
\begin{aligned}
& \left|L_{n}(z)-L_{n+1}(z)\right|=\left|L_{n}(z)-\left(L_{n}\right)_{\left[\lambda_{n}\right]}^{\left[\gamma_{n}\right]}(z)\right| \\
& \quad \leqslant \frac{|z|}{\delta}\left|\frac{1}{\lambda_{n}}-\frac{1}{\gamma_{n}}\right| \cdot A_{\varepsilon} \exp \left(\left(h_{L}(\theta)+\frac{2 \varepsilon}{3}\right) r\right), \quad z=r e^{i \theta}, r \geqslant 0, n \in \mathbb{N} .
\end{aligned}
$$

Отсюда левая часть (2.7) оценивается сверху как

$$
\begin{aligned}
& \left|L_{n}(z)-G(z)\right| \leqslant \sum_{k=n}^{\infty}\left|L_{k}(z)-L_{k+1}(z)\right| \\
& \quad \leqslant A_{\varepsilon} \sum_{k=n}^{\infty}\left|\frac{1}{\lambda_{k}}-\frac{1}{\gamma_{k}}\right| \cdot \frac{r}{\delta} \exp \left(\left(h_{L}(\theta)+\frac{2 \varepsilon}{3}\right) r\right), \quad z=r e^{i \theta}, r \geqslant 0, n=1,2, \ldots,
\end{aligned}
$$

и при $C_{\varepsilon}=\left(A_{\varepsilon} / \delta\right) \sup _{r \geqslant 0}(r \exp (-\varepsilon r / 3))$ получаем оценку $(2.7)$.

\section{§ 3. Оценка сверху многочленов}

Пусть $\Lambda$-последовательность в $\mathbb{C}, 0 \notin \Lambda ; n_{\Lambda}(t)=\Lambda(\bar{D}(t))$ - считающая функuия последовательности $\Lambda$, где $\bar{D}(t) \subset \mathbb{C}$ - замкнутый круг радиуса $t$ с центром в нуле. При этом полагаем, что

$$
\bar{\Delta}_{\Lambda}=\limsup _{t \rightarrow+\infty} \frac{n_{\Lambda}(t)}{t}, \quad N_{\Lambda}(t)=\int_{0}^{t} \frac{n_{\Lambda}(t)}{t} d t, \quad \bar{p}_{\Lambda}=\limsup _{t \rightarrow+\infty} \frac{N_{\Lambda}(t)}{t}
$$

- соответственно верхняя плотность, усредненная (проинтегрированная) считающая функиия и верхняя усредненная плотность последовательности $\Lambda$ (см. $[18 ; \mathrm{I} .1])$.

Легко устанавливаются неравенства (см. [13; с. 18], [18; I.1])

$$
\bar{p}_{\Lambda} \leqslant \bar{\Delta}_{\Lambda} \leqslant e \bar{p}_{\Lambda}
$$

ПРЕДЛОЖЕНИЕ 3.1. Пусть $\Lambda$ - последовательность конечной верхней плотности $\bar{\Delta}_{\Lambda}, 0 \notin \Lambda$. Тогда при любом $\varepsilon>0$ найдутся постоянные $C_{\varepsilon} \geqslant 0 u$ $T_{\varepsilon} \geqslant 0$ такие, что для интеграла

$$
I_{\Lambda}(r ; T) \stackrel{\text { def }}{=} \int_{0}^{T} \ln \left(1+\frac{r}{t}\right) d n_{\Lambda}(t)
$$

при всех $r>0$ и $T \geqslant T_{\varepsilon}$ справедлива оценка

$$
I_{\Lambda}(r ; T) \leqslant\left(\bar{\Delta}_{\Lambda}+\varepsilon\right) T \ln \left(1+\frac{r}{T}\right)+\left(\bar{p}_{\Lambda}+\varepsilon\right) r \ln \left(1+\frac{T}{r}\right)+C_{\varepsilon} .
$$


ДОКАЗАТЕЛЬСТво. Используя интегрирование по частям, получаем

$$
I_{\Lambda}(r ; T) \leqslant \int_{0}^{T} \ln \left(1+\frac{r}{t}\right) d n_{\Lambda}(t)=n_{\Lambda}(T) \ln \left(1+\frac{r}{T}\right)+\int_{0}^{T} \frac{r}{r+t} \frac{n_{\Lambda}(t)}{t} d t,
$$

где последний интеграл равен

Отсюда

$$
\int_{0}^{T} \frac{r}{r+t} d N_{\Lambda}(t)=\frac{r N_{\Lambda}(T)}{r+T}+\int_{0}^{T} \frac{r N_{\Lambda}(t)}{(r+t)^{2}} d t
$$

$$
I_{\Lambda}(r ; T) \leqslant n_{\Lambda}(T) \ln \left(1+\frac{r}{T}\right)+\frac{r N_{\Lambda}(T)}{r+T}+\int_{0}^{T} \frac{r N_{\Lambda}(t)}{(r+t)^{2}} d t, \quad r \geqslant 0 .
$$

В силу (3.2) и конечности верхней плотности $\bar{\Delta}_{\Lambda}$ усредненная верхняя плотность $\bar{p}_{\Lambda}$ также конечна. Значит, при любом $\varepsilon>0$ найдется постоянная $c_{\varepsilon} \geqslant 0$ такая, что

$$
n_{\Lambda}(t) \leqslant\left(\bar{\Delta}_{\Lambda}+\frac{\varepsilon}{2}\right) t+c_{\varepsilon}, \quad N_{\Lambda}(t) \leqslant\left(\bar{p}_{\Lambda}+\varepsilon\right) t+c_{\varepsilon}, \quad t \geqslant 0 .
$$

Применение (3.6) к (3.5) дает оценку

$$
\begin{aligned}
I_{\Lambda}(r ; T) \leqslant & \left(\left(\bar{\Delta}_{\Lambda}+\frac{\varepsilon}{2}\right) T+c_{\varepsilon}\right) \ln \left(1+\frac{r}{T}\right)+r \frac{\left(\bar{p}_{\Lambda}+\varepsilon\right) T+c_{\varepsilon}}{r+T} \\
& +r \int_{0}^{T} \frac{\left(\bar{p}_{\Lambda}+\varepsilon\right) t+c_{\varepsilon}}{(r+t)^{2}} d t .
\end{aligned}
$$

Выгислим последний интеграл в правой части (3.7):

$$
\begin{gathered}
\int_{0}^{T} \frac{\left(\bar{p}_{\Lambda}+\varepsilon\right) t+c_{\varepsilon}}{(r+t)^{2}} d t=\left(\bar{p}_{\Lambda}+\varepsilon\right) \int_{0}^{T} \frac{t d t}{(r+t)^{2}}+c_{\varepsilon} \int_{0}^{T} \frac{d t}{(r+t)^{2}} \\
=\left(\bar{p}_{\Lambda}+\varepsilon\right) \int_{0}^{T} t d\left(-\frac{1}{(r+t)}\right)+c_{\varepsilon}\left(\frac{1}{r}-\frac{1}{r+T}\right) \\
=\left(-\left(\bar{p}_{\Lambda}+\varepsilon\right) \frac{T}{r+T}+\int_{0}^{T} \frac{d t}{(r+t)}\right)+c_{\varepsilon} \frac{T}{r(r+T)} \\
=-\frac{\left(\bar{p}_{\Lambda}+\varepsilon\right) T}{r+T}+\left(\bar{p}_{\Lambda}+\varepsilon\right) \ln \left(1+\frac{T}{r}\right)+\frac{c_{\varepsilon} T}{r(r+T)} .
\end{gathered}
$$

Подставляя результат в правую часть (3.7), получаем

$$
\begin{aligned}
I_{\Lambda}(r ; T) \leqslant & \left(\bar{\Delta}_{\Lambda}+\frac{\varepsilon}{2}\right) T \ln \left(1+\frac{r}{T}\right)+c_{\varepsilon} \ln \left(1+\frac{r}{T}\right) \\
& +r \frac{c_{\varepsilon}}{r+T}+r\left(\bar{p}_{\Lambda}+\varepsilon\right) \ln \left(1+\frac{T}{r}\right)+\frac{c_{\varepsilon} T}{r(r+T)} .
\end{aligned}
$$

При $T \geqslant 2 c_{\varepsilon} / \varepsilon$ сумма первых двух слагаемых в правой части оценивается сверху величиной

$$
\left(\bar{\Delta}_{\Lambda}+\varepsilon\right) T \ln \left(1+\frac{r}{T}\right)+\left(c_{\varepsilon}-\frac{\varepsilon T}{2}\right) \ln \left(1+\frac{r}{T}\right) \leqslant\left(\bar{\Delta}_{\Lambda}+\varepsilon\right) T \ln \left(1+\frac{r}{T}\right) .
$$

Отсюда согласно (3.8) получаем

$$
I_{\Lambda}(r ; T) \leqslant\left(\bar{\Delta}_{\Lambda}+\varepsilon\right) T \ln \left(1+\frac{r}{T}\right)+\left(\bar{p}_{\Lambda}+\varepsilon\right) r \ln \left(1+\frac{T}{r}\right)+2 c_{\varepsilon},
$$

что при $T \geqslant T_{\varepsilon}=2 c_{\varepsilon} / \varepsilon$ и $C_{\varepsilon}=2 c_{\varepsilon}$ дает требуемую оценку (3.4). 
ПРЕДЛОЖЕНИЕ 3.2. Пусть $0<d<+\infty$, а $\Lambda$ - последовательность конечной верхней плотности $\bar{\Delta}_{\Lambda}, 0 \notin \Lambda$. Тогда при любом $\varepsilon>0$ найдутся постоянные $C_{\varepsilon} \geqslant 0$ и $T_{\varepsilon} \geqslant 0$ такие, что при всех $T \geqslant T_{\varepsilon}$ справедлива оченка

$$
\sup _{r \geqslant 0}\left(I_{\Lambda}(r ; T)-d r\right) \leqslant T \cdot b\left(\bar{\Delta}_{\Lambda}+\varepsilon, \bar{p}_{\Lambda}+\varepsilon, d\right)+C_{\varepsilon},
$$

əде $\partial \Omega я \Delta, p \geqslant 0 u d>0$

$$
0 \leqslant b(\Delta, p, d) \stackrel{\text { def }}{=} \sup _{x>0}\left(\Delta \ln (1+x)+p x \ln \left(1+\frac{1}{x}\right)-d x\right)<+\infty
$$

ДокАЗАТЕЛЬСТво. Соотношение (3.9) сразу следует из предложения 3.1, если после выгитания из $I_{\Lambda}(r ; T)$ величины $d r$ в $(3.4)$ и применения к обеим частям полученного неравенства операции $\sup _{r \geqslant 0}$ использовать замену $x=r / T$ в правой части.

В связи с оценкой (3.9) нам потребуются достаточно элементарные оценки и свойства величины $b(\Delta, p, d)$ из $(3.10)$, которые дает следуюшее

ПРЕДЛОЖЕНИЕ 3.3. Функция $b(\cdot, \cdot, \cdot)$ является непрерывной по каждой из трех переменных, не убывающей по двум первым переменныц и невозрастающей по последней переменной. При (ср. с (3.2))

$$
p \leqslant \Delta \leqslant e p
$$

в обозначениях $a^{+}=\max \{a, 0\}, \ln ^{+} x=\max \{\ln x, 0\}$ справедливы оценки

$$
\begin{aligned}
b(\Delta, p, d) & \leqslant p \frac{\Delta}{d} \ln \left(1+\frac{d}{\Delta}\right)+\Delta \ln ^{+} \frac{\Delta}{d}-(\Delta-d)^{+} \\
& \leqslant p+\Delta \ln ^{+} \frac{\Delta}{d} \\
& \leqslant p+e p \ln ^{+} \frac{e p}{d} .
\end{aligned}
$$

ДокАЗАТЕЛЬСтво. Доказательства свойств функции $b$, касающиеся ееповедения по каждой переменной, опускаем как элементарные. Установим оценки (3.12).

Непрерывная на $(0,+\infty)$ функция

$$
m(x) \stackrel{\text { def }}{=} \Delta \ln (1+x)+p x \ln \left(1+\frac{1}{x}\right)-d x, \quad x>0,
$$

продолженная по непрерывности значением $m(0)=0$ в точку 0 , стремится к $-\infty$ при $x \rightarrow+\infty$, т.е. достигает своего максимального значения на $[0,+\infty)$. В частности, выполнены неравенства (3.10). 
Пусть $x_{m}$ - точка максимума функции $m(x)$ на $[0,+\infty)$. Тогда либо $x_{m}=0$, либо число $x_{m}$ удовлетворяет уравнению

$$
m^{\prime}(x)=\frac{\Delta-p}{1+x}+p \ln \left(1+\frac{1}{x}\right)-d=0, \quad x>0 .
$$

При $p \leqslant \Delta$ имеем

$$
m^{\prime \prime}(x)=-\left(\frac{\Delta-p}{(1+x)^{2}}+\frac{p}{x(1+x)}\right)<0
$$

для всех $x>0$, т.е. функция $m^{\prime}(x)$ - строго монотонно убываюшая на $(0,+\infty)$. Кроме того, $\lim _{x \rightarrow 0} m^{\prime}(x)=+\infty$ и $\lim _{x \rightarrow+\infty} m^{\prime}(x)=-d<0$. Следовательно, уравнение (3.14) имеет единственное положительное решение, которое и будет точкой максимума $x_{m}$. При этом

$0=\frac{\Delta-p}{1+x_{m}}+p \ln \left(1+\frac{1}{x_{m}}\right)-d \leqslant \frac{\Delta-p}{1+x_{m}}+\frac{p}{x_{m}}-d=\frac{\Delta x_{m}+p}{\left(1+x_{m}\right) x_{m}}-d \leqslant \frac{\Delta}{x_{m}}-d$,

т.е. $x_{m} \leqslant \Delta / d$ при $p \leqslant \Delta$. Отсюда и из определений (3.13) и (3.10) при условии $p \leqslant \Delta$ в силу возрастания функции $x \ln (1+1 / x), x>0$, получаем

$$
\begin{aligned}
b(\Delta, p, d) & =m\left(x_{m}\right)=p x_{m} \ln \left(1+\frac{1}{x_{m}}\right)+\left(\Delta \ln \left(1+x_{m}\right)-d x_{m}\right) \\
& \leqslant p \frac{\Delta}{d} \ln \left(1+\frac{d}{\Delta}\right)+\max _{x \geqslant 0}(\Delta \ln (1+x)-d x) .
\end{aligned}
$$

Если $\Delta / d \geqslant 1$, то последний максимум здесь достигается в точке $x=\Delta / d-1=$ $(\Delta / d-1)^{+}$, а в противном случае - в точке $x=0=(\Delta / d-1)^{+}$. Таким образом,

$$
b(\Delta, p, d) \leqslant p \frac{\Delta}{d} \ln \left(1+\frac{d}{\Delta}\right)+\Delta \ln \left(1+\left(\frac{\Delta}{d}-1\right)^{+}\right)-d\left(\frac{\Delta}{d}-1\right)^{+} .
$$

Правая часть здесь совпадает с правой частью неравенства (3.12a), продолжение - (3.12b) - использует элементарное неравенство $x \ln (1+1 / x) \leqslant 1$, а дальнейшее - (3.12c) - следует из правой части условия (3.11).

Перенесем последние результаты на многочлены, нормированные в нуле значением 1.

ПРЕДЛОЖЕНИЕ 3.4. Пусть $\Lambda=\left\{\lambda_{n}\right\}$ - последовательность конечной верхней плотности, $0 \notin \Lambda$, и число $d>0$. Если $\bar{\Delta}_{\Lambda}<\bar{\Delta} u \bar{p}_{\Lambda}<\bar{p}$, то найдется постоянная $C_{d}>0$ такая, что при любом $n$ для всех $z \in \mathbb{C}$ справедлива оценка

$$
\left|\prod_{k=1}^{n}\left(1-\frac{z}{\lambda_{k}}\right)\right| \leqslant C_{d} \exp \left(b(\bar{\Delta}, \bar{p}, d) \max _{1 \leqslant k \leqslant n}\left|\lambda_{k}\right|\right) \cdot \exp (d|z|) .
$$

Ослабляя, вообще говоря, әту оценку, можно заменить в правой части (3.15) величину $b(\bar{\Delta}, \bar{p}, d)$, определенную в (3.10), на правую часть в неравенствах (3.12a), (3.12b) или (3.12c) $c \Delta=\bar{\Delta}$ и $p=\bar{p}$. 
ДокАЗАТЕЛЬСТВо. Положим $T_{n}=\max _{1 \leqslant k \leqslant n}\left|\lambda_{k}\right|$. Тогда в обозначении (3.3) при $r=|z|$ имеем

$$
\sum_{k=1}^{n} \ln \left|1-\frac{z}{\lambda_{k}}\right| \leqslant \sum_{k=1}^{n} \ln \left(1+\frac{r}{\left|\lambda_{k}\right|}\right) \leqslant I_{\Lambda}\left(r ; T_{n}\right) .
$$

Отсюда оценка (3.9) предложения 3.2 влечет за собой то, что при достаточно малом $\varepsilon>0$, для которого $\bar{\Delta}_{\Lambda}+\varepsilon<\bar{\Delta}_{\text {и }} \bar{p}_{\Lambda}+\varepsilon<\bar{p}$, найдутся постоянные $C_{\varepsilon}$ и $T_{\varepsilon}$ такие, что при всех достаточно больших $n$, для которых $T_{n} \geqslant T_{\varepsilon}$, справедлива оценка

$$
\begin{aligned}
\sup _{|z| \geqslant 0}\left(\sum_{k=1}^{n} \ln \left|1-\frac{z}{\lambda_{k}}\right|-d|z|\right) & \leqslant T_{n} \cdot b\left(\bar{\Delta}_{\Lambda}+\varepsilon, \bar{p}_{\Lambda}+\varepsilon, d\right)+C_{\varepsilon} \\
& \leqslant \max _{1 \leqslant k \leqslant n}\left|\lambda_{k}\right| \cdot b(\bar{\Delta}, \bar{p}, d)+C_{\varepsilon} .
\end{aligned}
$$

Увеличивая, если необходимо, постоянную $C_{\varepsilon}$, распространяем последнюю оценку на все $n$. Ее потенцирование дает (3.15). Заключительное замечание об ослабленных вариантах оценки (3.15) - это очевидное следствие неравенств (3.12) предложения 3.3.

Версию (3.12c) неравенств (3.12) предложения 3.3 дополняет

ПРЕДЛОЖЕНИЕ 3.5. Пусть иисло $d>0, D-$ выпуклая область в $\mathbb{C}$ периметра $p=p(D)<\infty$ и $\Lambda=\left\{\lambda_{n}\right\}$ - последовательность неединственности для $D, 0 \notin \Lambda$. Тогда справедливо неравенство

$$
b\left(\bar{\Delta}_{\Lambda}, \bar{p}_{\Lambda}, d\right)<\frac{p}{2 \pi}\left(1+e \ln ^{+} \frac{e p}{2 \pi d}\right) .
$$

ДоКАЗАТЕЛЬСТво. В силу (3.2) по предложению 3.3 из заключительной оценки (3.12c) цепочки неравенств (3.12) следует неравенство

$$
b\left(\bar{\Delta}_{\Lambda}, \bar{p}_{\Lambda}, d\right)<\bar{p}_{\Lambda}+e \bar{p}_{\Lambda} \ln ^{+} \frac{e \bar{p}_{\Lambda}}{d} .
$$

Далее используется элементарная

Лемма 3.1. Верхняя усредненная плотность $\bar{p}_{\Lambda}$ последовательности неединственности $\Lambda, 0 \notin \Lambda$, для выпуклой области $D$ периметра р строго меньше чем $p /(2 \pi)$.

ДоКАЗАТЕЛЬСТво ЛЕмМЫ. По определению последовательности неединственности для $D$ найдется функция $L \in P_{D^{*}}$ такая, что $L \not \equiv 0$ и $L(\Lambda)=0$. Не умаляя обшности, можно считать, что $L(0)=1$. По определению $(3.1)$ и по классической формуле Йенсена для $L$ имеем

$$
\begin{aligned}
\bar{p}_{\Lambda} \leqslant \limsup _{t \rightarrow+\infty} \frac{N_{\text {Zero }_{L}}(t)}{t} & =\limsup _{t \rightarrow+\infty} \frac{1}{2 \pi t} \int_{0}^{2 \pi} \ln \left|L\left(t e^{i \theta}\right)\right| d \theta \leqslant \frac{1}{2 \pi} \int_{0}^{2 \pi} h_{L}(\theta) d \theta \\
& <\frac{1}{2 \pi} \int_{0}^{2 \pi} K_{D^{*}}(\theta) d \theta .
\end{aligned}
$$

Последний интеграл, как известно [12; гл. I, § 19], [19; гл. I, §2], есть периметр области $D^{*}$, т.е. равен $p$, что и требовалось.

По лемме 3.1 правая часть в (3.17) увеличится, если заменить в ней верхнюю усредненную плотность $\bar{p}_{\Lambda}$ на бо́льшую величину $p /(2 \pi)$. 


\section{§4. Вторая аппроксимационная теорема}

Следующая аппроксимационная теорема напрямую нацелена на доказательство основной теоремы.

ТЕорема 2 (аппроксимационная). Пусть иисло $d>0, L \not \equiv 0-$ иелая функиия әкспоненциального типа $u \Lambda=\left\{\lambda_{n}\right\} \subset \operatorname{Zero}_{L}, 0 \notin \Lambda, \bar{\Delta}_{\Lambda} u \bar{p}_{\Lambda}$-конечные верхняя и верхняя усредненная плотности последовательности $\Lambda$ соответственно. Если для некоторой последовательности $\Gamma=\left\{\gamma_{n}\right\} \subset \mathbb{C}, 0 \notin \Gamma$, выполнено соотношение

$$
\liminf _{n \rightarrow \infty} \frac{\ln \left(\sum_{k \geqslant n}\left|1 / \lambda_{k}-1 / \gamma_{k}\right|\right)}{\max _{k \leqslant n}\left|\lambda_{k}\right|}<-b\left(\bar{\Delta}_{\Lambda}, \bar{p}_{\Lambda}, d\right)
$$

әде правая часть определена равенством (3.10), то найдется иелая функиия экспоненииального типа $G \neq \equiv, G(0)=L(0)$, с индикатором роста $h_{G}=h_{L}$ и с последовательностью нулей $\operatorname{Zero}_{G}=\left(\operatorname{Zero}_{L} \backslash \Lambda\right) \cup \Gamma$ такая, что некоторая подпоследовательность $\left\{M_{n_{k}}\right\}, k=1,2, \ldots$, последовательности иельх функицй әкспоненицального типа

$$
M_{n}(z)=\prod_{k=1}^{n}\left(1-\frac{z}{\gamma_{k}}\right) \cdot L(z)-\prod_{k=1}^{n}\left(1-\frac{z}{\lambda_{k}}\right) \cdot G(z), \quad n=1,2, \ldots,
$$

стремится $к$ тождественному нулю равномерно на компактах из $\mathbb{C}$ и найдется постоянная $C \geqslant 0$, к которой выполнены равномерные по $k$ оценки

$$
\left|M_{n_{k}}(z)\right| \leqslant C \exp \left(\left(h_{L}(\theta)+d\right) r\right), \quad z=r e^{i \theta} \in \mathbb{C}, \quad r \geqslant 0, \quad k \in \mathbb{N} .
$$

ДокАЗАТЕЛЬСтво. Выполнены все условия теоремы 1. В частности, ряд (2.5) сходится в силу условия (4.1). В качестве функции $G$ выбираем предел канонической последовательности (2.4) для функции $L$ целых функций экспоненциального типа $\left\{L_{n}\right\}$, сдвигающей подпоследовательность нулей $\Lambda$ в последовательность Г из заключения теоремы 1 . Тогда с учетом теоремы 1 остается доказать только сушествование подпоследовательности $\left\{M_{n_{k}}\right\}, k=1,2, \ldots$, последовательности (4.2) с требуемыми свойствами.

Положим

$$
R_{n}=\sum_{k=n}^{\infty}\left|\frac{1}{\lambda_{k}}-\frac{1}{\gamma_{k}}\right|, \quad T_{n}=\max _{1 \leqslant k \leqslant n}\left|\lambda_{k}\right|, \quad n=1,2, \ldots
$$

Функция $b(\cdot, \cdot, \cdot)$, определенная равенством $(3.10)$, как отмечалось в предложении 3.3 , непрерывна по каждой из трех переменных. Поэтому существуют числа $\bar{\Delta}, \bar{p}, \bar{d}>0$ такие, что

$$
\bar{\Delta}_{\Lambda}<\bar{\Delta}, \quad \bar{p}_{\Lambda}<\bar{p}, \quad \bar{d}<d,
$$

а условие (4.1) сохраняется и после замены в правой части $\bar{\Delta}_{\Lambda}, \bar{p}_{\Lambda}$ и $d$ соответственно на $\bar{\Delta}, \bar{p}$ и $\bar{d}$. В этом случае в обозначениях (4.4) условие (4.1) означает, 
что найдется подпоследовательность $\left\{n_{k}\right\}, k=1,2, \ldots$, натуральных чисел, для которых

$$
R_{n_{k}} \leqslant C_{0} \exp \left(-b(\bar{\Delta}, \bar{p}, \bar{d}) T_{n_{k}}\right)
$$

где постоянная $C_{0}$ не зависит от $k$. Выберем число $\varepsilon>0$ столь малым, что

$$
\bar{d}<\bar{d}+\varepsilon \leqslant d
$$

Каждая целая функция экспоненциального типа $M_{n}$, определенная в $(4.2)$, представима в виде

$$
M_{n}(z)=\prod_{k=1}^{n}\left(1-\frac{z}{\lambda_{k}}\right) \cdot\left(\prod_{k=1}^{n} \frac{1-z / \gamma_{k}}{1-z / \lambda_{k}} L(z)-G(z)\right), \quad n \in \mathbb{N}
$$

Здесь в последней скобке стоит разность $L_{n}-G$, где $\left\{L_{n}\right\}$ - упоминавшаяся выше каноническая последовательность для $L$, сдвигающая $\Lambda$ в $Г$. Таким образом, для всех $z \in \mathbb{C}$ справедлива оценка

$$
\left|M_{n}(z)\right| \leqslant\left|\prod_{k=1}^{n}\left(1-\frac{z}{\lambda_{k}}\right)\right| \cdot\left|L_{n}(z)-G(z)\right|, \quad n=1,2, \ldots
$$

Ввиду сходимости последовательности $\left\{L_{n}\right\}$ к $G$ это обеспечивает пока, как минимум, поточечную сходимость последовательности $\left\{M_{n}\right\}$ к тождественному нулю.

Из (4.8) по аппроксимационной теореме 1 из оценки скорости сходимости (2.7) для некоторой постоянной $C_{\varepsilon}$ при всех $z=r e^{i \theta} \in \mathbb{C}$ получаем

$$
\left|M_{n}(z)\right| \leqslant\left|\prod_{k=1}^{n}\left(1-\frac{z}{\lambda_{k}}\right)\right| \cdot C_{\varepsilon} R_{n} \exp \left(\left(h_{L}(\theta)+\varepsilon\right) r\right), \quad n \in \mathbb{N} .
$$

Кроме того, из соотношений (4.5) по предложению 3.4 найдется постоянная $C_{\bar{d}}$ такая, что выполнена оценка (3.15) с $\bar{d}$ вместо $d$. Эта оценка вместе с (4.9) дает оценку

$$
\begin{gathered}
\left|M_{n}(z)\right| \leqslant C_{\bar{d}} \exp \left(b(\bar{\Delta}, \bar{p}, \bar{d}) T_{n}\right) \cdot C_{\varepsilon} R_{n} \exp \left(\left(h_{L}(\theta)+\bar{d}+\varepsilon\right) r\right) \\
z=r e^{i \theta} \in \mathbb{C}, \quad n=1,2, \ldots
\end{gathered}
$$

Переходя здесь к подпоследовательности $\left\{M_{n_{k}}\right\}$, из (4.6) получаем

$$
\begin{aligned}
\left|M_{n_{k}}(z)\right| \leqslant & C_{\bar{d}} \exp \left(b(\bar{\Delta}, \bar{p}, \bar{d}) T_{n_{k}}\right) \\
& \times C_{\varepsilon} C_{0} \exp \left(-b(\bar{\Delta}, \bar{p}, \bar{d}) T_{n_{k}}\right) \cdot \exp \left(\left(h_{L}(\theta)+\bar{d}+\varepsilon\right) r\right) \\
= & C_{\bar{d}} C_{\varepsilon} C_{0} \exp \left(\left(h_{L}(\theta)+\bar{d}+\varepsilon\right) r\right), \quad z=r e^{i \theta} \in \mathbb{C}, \quad k=1,2, \ldots
\end{aligned}
$$

Отсюда согласно условию (4.7) на выбор $\bar{d}$ при $C=C_{\bar{d}} C_{\varepsilon} C_{0}$ следуют равномерные по $k$ оценки (4.3). Из поточечной сходимости последовательности $\left\{M_{n_{k}}\right\}$ к нулю и равномерных по $k$ оценок (4.3) по теореме Витали о сходимости [20; 5.2.1] последовательность функций $\left\{M_{n_{k}}\right\}$ сходится к нулю равномерно на компактах из $\mathbb{C}$. 


\section{§5. Доказательства основной теоремы и следствия}

Используются сведения, подробно изложенные в работах [1], [2], [6], [17].

Пусть $\Omega$ - ограниченная вьпуклая область в $\mathbb{C}$. Преобразование Лапласа $(0.3)$ осушествляет изоморфизм сопряженного пространства $H^{*}$ на пространства целых функций экспоненциального типа $P$ и индуцирует на $P$ топологию сильно сопряженного пространства $H^{*}$. Можно дать и внутреннее определение этой индуцированной топологии как некоторой естественной топологии индуктивного предела на $P$. Отсылая за деталями к [1], [2], отметим здесь лишш условия сходимости последовательности: последовательность $\left\{F_{n}\right\} \subset P$ сходится в топологии $P$, если и только если она сходится равномерно на компактах в $\mathbb{C}$ и одновременно для некоторой тригонометрически выпуклой функции $h<K_{\Omega^{*}}$ существует постоянная $C$ такая, что справедливь равномерные по п оценки $\left|F_{n}(z)\right| \leqslant C \exp (h(\theta) r), z=r e^{i \theta} \in \mathbb{C}, r \geqslant 0, n=1,2, \ldots$.

Следуя [1], [2], для подпространства $W \subset H$ подпространство всех функционалов из $H^{*}$, аннулирующих $W$, обозначаем через $W^{0}$, а подпространство в $P$ всех преобразований Лапласа (0.3), т.е. характеристических функций, функционалов из $W^{0}$ обозначаем $T\left(W^{0}\right)$.

В основе доказательства приведенной ниже теоремы 3 и основной теоремы лежит следуюшее основополагающее для задачи о пересечении инвариантных подпространств

ПРЕДЛОЖЕНИЕ 5.1 [2; предложение 6.2]. Пусть инвариантные подпространства $W_{1}, \ldots, W_{n}$ в $H$ допускают спектральный синтез. Пересечение $\bigcap_{k=1}^{n} W_{k}$ допускает спектральный синтез тогда и только тогда, когда для любого набора $\bar{\alpha}=\left(\alpha_{1}, \ldots, \alpha_{n}\right)$ комплексных чисел, связанных условием $\alpha_{1}+\cdots+\alpha_{n}=0$, и какой-либо точки $\lambda \in \mathbb{C}$, не принадлежсащей спектру ни одного из пространств $W_{k}, k=1, \ldots, n$, замыкание в $P$ множества

$$
\mathscr{T}(\bar{\alpha}, \lambda) \stackrel{\text { def }}{=}\left\{F=F_{1}+\cdots+F_{n}: F_{k} \in T\left(W_{k}^{0}\right), F_{k}(\lambda)=\alpha_{k}, 1 \leqslant k \leqslant n\right\}
$$

содержит тождественный нуль.

В этом параграфе из последнего предложения потребуется только достаточность в следующей форме (ср. [2; предложение 6.4]).

ПРЕДЛОЖЕНИЕ 5.2. Пусть $W_{1}, W_{2}$ - инвариантные подпространства в $H$, допускающие спектральный синтез, и $0 \notin \operatorname{Spec} W_{1} \cap \operatorname{Spec} W_{2}$. Если для цельх функций $L \in T\left(W_{1}^{0}\right)$ и $G \in T\left(W_{2}^{0}\right)$ с условием $L(0)=G(0)=1$ найдутся последовательности многочленов $\left\{g_{n}\right\}$ u $\left\{l_{n}\right\}$ такие, что $g_{n}(0)=l_{n}(0)=1$, a последовательность $g_{n} L-l_{n} G$ стремится к тождественному нулю в $P$, то инвариантное подпространство $W_{1} \cap W_{2}$ также допускает спектральный синтез.

ДокаЗАТЕЛЬство. Пространство $P$ естественным образом наделяется структурой топологического модуля над кольцом $\mathbb{C}[z]$. При этом подпространства $T\left(W_{1}^{0}\right), T\left(W_{2}^{0}\right)$ образуют замкнутые подмодули в топологическом модуле $P$ над $\mathbb{C}[z]$. Значит, $g_{n} L \in T\left(W_{1}^{0}\right)$ и $l_{n} G \in T\left(W_{1}^{0}\right)$ при любом $n=1,2, \ldots$. Отсюда 
множество $T(\bar{\alpha}, 0)$ из (5.1) при $n=2, \bar{\alpha}=(\alpha,-\alpha), \alpha \in \mathbb{C}$, включает в себя последовательность

$$
\left\{\alpha g_{n} L+\left(-\alpha l_{n} G\right): n=1,2, \ldots\right\}
$$

предел которой в $P$ - тождественный нуль. Остается воспользоваться предложением 5.1.

Пусть $B$ - выпуклое множество в $\mathbb{C}$, число $d>0$. Выпуклое множество

$$
B^{d} \stackrel{\text { def }}{=} \bigcup_{z \in B}\{\zeta \in \mathbb{C}:|\zeta-z|<d\}
$$

называем $^{2} d$-вздутием множества $B$. Для опорных функций множеств $B$ и его $d$-вздутия $B^{d}$ справедливо тождество [19; гл. I, $\left.\S 4\right]$

$$
K_{B^{d}}(\theta) \equiv K_{B}(\theta)+d, \quad \theta \in \mathbb{R}
$$

Теорема 3. Пусть $\Lambda=\left\{\lambda_{n}\right\}$ u $\Lambda^{\prime}, \quad \Gamma=\left\{\gamma_{n}\right\} u \Gamma^{\prime}$ - четыре последовательности в $\mathbb{C}, \bar{\Delta}_{\Lambda}$ и $\bar{p}_{\Lambda}$ - соответственно верхняя и верхняя усредненная плотности последовательности $\Lambda$, а последовательность $\Lambda \cup\left(\Lambda^{\prime} \triangle \Gamma^{\prime}\right)$ является последовательностью неединственности для некоторой выпуклой области $D \Subset \Omega$, m.е. $d=\operatorname{dist}(D, \partial \Omega)>0$. Если выполнено соотношение (4.1), то инвариантное подпространство $W\left(\Lambda \cup \Lambda^{\prime}\right) \cap W\left(\Gamma \cup \Gamma^{\prime}\right)$ в $H$ допускает спектральный синтез.

Заключение теоремы остается в силе, если заменить в правой части условия (4.1) величину $b\left(\bar{\Delta}_{\Lambda}, \bar{p}_{\Lambda}, d\right)($ см. (3.10)) на правую часть в неравенствах (3.12a), (3.12b) или (3.12c) c $\Delta=\bar{\Delta}_{\Lambda} u p=\bar{p}_{\Lambda}$.

ДокАЗАТЕльство. Условие на последовательность $\Lambda_{0} \stackrel{\text { def }}{=} \Lambda \cup\left(\Lambda^{\prime} \triangle \Gamma^{\prime}\right)$ эквивалентно существованию целой функции экспоненциального типа $L \not \equiv 0$, обращающейся в нуль на последовательности $\Lambda_{0}$, с индикатором роста $h_{L}(\theta)<K_{D^{*}}(\theta)$, $\theta \in \mathbb{R}$. Не ограничивая общности, можно считать (возможно, после сдвига $\mathbb{C}$ и последующего домножения функции $L$ на некоторую ненулевую константу) выполненными условия $0 \notin \Lambda \cup \Lambda^{\prime} \cup \Gamma \cup \Gamma^{\prime} \cup \operatorname{Zero}_{L}, L(0)=1$. Неравенство $h_{L}<K_{D^{*} \text { вмес- }}$ те со свойством $L\left(\Lambda_{0}\right)=0$ означает, что функционал $\mathscr{L} \in H^{*}(D) \subset H^{*}$ с характеристической функцией $L$ аннулирует систему экспонент $\operatorname{Exp}_{\Lambda_{0}}$, определенную как в $(0.1)$, а значит, и замыкание ее линейной оболочки $\overline{\left\langle\operatorname{Exp}_{\Lambda_{0}}\right\rangle}=W\left(\Lambda_{0}\right) \supset W(\Lambda \cup$ $\left.\Lambda^{\prime}\right)$. Таким образом, $L \in T\left(\left(W\left(\Lambda \cup \Lambda^{\prime}\right)\right)^{0}\right)$. Ввиду условия (4.1) по аппроксимационной теореме 2 найдется целая функция экспоненциального типа $G \not \equiv 0$, $G(0)=L(0)=1$, с индикатором роста $h_{G}=h_{L}$ и с последовательностью нулей

$$
\operatorname{Zero}_{G}=\left(\operatorname{Zero}_{L} \backslash \Lambda\right) \cup \Gamma \supset\left(\Lambda_{0} \backslash \Lambda\right) \cup \Gamma=\left(\Lambda^{\prime} \Delta \Gamma^{\prime}\right) \cup \Gamma \supset \Gamma \cup \Gamma^{\prime},
$$

и при этом выполнены все свойства последовательности целых функций экспоненциального типа (4.2) из заключения аппроксимационной теоремы 2.

\footnotetext{
${ }^{2} \mathrm{~B}$ теории вьпуклых множеств множество $B^{d}$ называют также множеством, параллельным множеству В на расстоянии $d[19 ;$ гл. I, §4].
} 
Равенство индикаторов роста $h_{G}=h_{L}$ вместе с (5.4) означает, что функционал $\mathscr{G} \in H^{*}(D) \subset H^{*}$ с характеристической функцией $G$ аннулирует систему экспонент $\operatorname{Exp}_{\Gamma \cup \Gamma^{\prime}}$, а значит, и подпространство $\overline{\left\langle\operatorname{Exp}_{\Gamma \cup \Gamma^{\prime}}\right\rangle}=W\left(\Gamma \cup \Gamma^{\prime}\right)$. Таким образом, $G \in T\left(\left(W\left(\Gamma \cup \Gamma^{\prime}\right)\right)^{0}\right)$.

Введем в рассмотрение многочлены

$$
l_{n}(z)=\prod_{k=1}^{n}\left(1-\frac{z}{\lambda_{k}}\right), \quad g_{n}(z)=\prod_{k=1}^{n}\left(1-\frac{z}{\gamma_{k}}\right), \quad l_{n}(0)=g_{n}(0)=1 .
$$

В этих обозначениях последовательность (4.2) есть последовательность $g_{n} L-l_{n} G$. По аппроксимационной теореме 2 некоторая подпоследовательность $\left\{M_{n_{k}}\right\}$ последовательности функций $M_{n}=g_{n} L-l_{n} G$ стремится к тождественному нулю равномерно на компактах из $\mathbb{C}$ и для этой подпоследовательности выполнены равномерные по $k$ оценки (4.3). Из неравенства $h_{L}<K_{D^{*}}$ согласно (5.2) и (5.3) следует $h_{L}+d<K_{D^{*}}+d=K_{\left(D^{d}\right)^{*}}$, где $D^{d}-d$-вздутие выпуклой области. По описанию сходимости последовательностей в пространствах типа $P$ последние два предложения означают, что подпоследовательность $\left\{M_{n_{k}}\right\}$ сходится к тождественному нулю в пространстве $P_{\left(D^{d}\right)^{*}}$. Тем более она сходится к нулю в пространстве $P$, поскольку по определению числа $d=\operatorname{dist}(D, \partial \Omega)$ имеет место включение $D^{d} \subset \Omega$. Таким образом, последовательность функций $M_{n_{k}}=g_{n_{k}} L-l_{n_{k}} G, k=1,2, \ldots$, $L(0)=G(0)=l_{n_{k}}(0)=g_{n_{k}}(0)=1$, стремится к нулю в $P$. Отсюда по предложению 5.2 пересечение $W\left(\Lambda \cup \Lambda^{\prime}\right) \cap W\left(\Gamma \cup \Gamma^{\prime}\right)$ допускает спектральный синтез на $\Omega$.

Заключительное замечание об усилении условия (4.1) является очевидным следствием неравенств (3.12) предложения 3.3.

ДОКАЗАТЕЛЬСТво ОСНОвНОЙ ТЕОРЕМЫ. Согласно оценке (3.16) предложения 3.5 условие (1.4) основной теоремы влечет условие (4.1) теоремы 3 , из которой сразу следует основная теорема.

ДОКАЗАТЕЛЬСТво СЛЕДСТВИЯ. В силу условия (1.4) ряд (2.5) сходится. В качестве требуемой функции $G$ по теореме 1 можно выбрать предел в $P$ канонической последовательности функций, сдвигающей подпоследовательность нулей $\Lambda \subset \operatorname{Zero}_{L}$ в последовательность $\Gamma$.

Пусть $\mathscr{L}$ и $\mathscr{G}$ - функционалы с характеристическими функциями $L$ и $G$ соответственно. Оба эти функционала принадлежат $H^{*}(D)$, поскольку $h_{G}=h_{L}<$ $K_{D^{*}}$. Каждое из пространств $W_{\mathscr{L}}$ и $W \mathscr{G}$ решений однородных уравнений свертки $\mathscr{L} * f=0$ и $\mathscr{G} * f=0$ соответственно допускает спектральный синтез, и спектры их соответственно $\operatorname{Zero}_{L}=\Lambda \cup \operatorname{Spec} W$ и Zero $_{G}=\Gamma \cup \operatorname{Spec} W$. Следовательно,

$$
W_{\mathscr{L}}=W(\Lambda \cup \operatorname{Spec} W), \quad W_{\mathscr{G}}=W(\Gamma \cup \operatorname{Spec} W) .
$$

Отсюда имеет место равенство

$$
W(\Lambda \cup \operatorname{Spec} W) \cap W(\Gamma \cup \operatorname{Spec} W)=W_{\mathscr{L}} \cap W \mathscr{G},
$$

где правая часть - пространство решений системы (1.5). Применяя основную теорему при $\Lambda^{\prime}=\Gamma^{\prime}=$ Spec $W$, убеждаемся, что инвариантное подпространство в левой части (5.5) допускает спектральньй синтез. Его спектр совпадает с пересечением $(\Lambda \cup \operatorname{Spec} W) \cap(\Gamma \cup \operatorname{Spec} W)=\operatorname{Spec} W$, поскольку последовательности $\Lambda$ и $\Gamma$ 
не пересекаются. Два допускающих спектральный синтез инвариантных подпространства с одинаковыми спектрами совпадают, значит, левая часть в $(5.5)$ - это в точности подпространство $W$.

ЗАмЕчАниЕ. Еще более заостряя формулировку следствия в отношении конструктивности, подчеркнем, что согласно его доказательству функция $G$ строится по функции $L$ в явном виде - как предел конкретной последовательности (2.4). Более того, можно вьписать разложение Адамара для функции $G$, пользуясь разложением Адамара функции $L$ (точное выражение см. в $[11 ; \S 1$, п. $6,(1.37)]$ ).

\section{§6. Один пример}

В этом параграфе для определенных выпуклых областей $\Omega$ строится пример последовательностей неединственности $\Lambda=\left\{\lambda_{n}\right\}$ и $\Gamma=\left\{\gamma_{n}\right\}$ для $\Omega$, скорость сближения которых задается оценкой

$$
\liminf _{n \rightarrow \infty} \frac{\ln \left(\sum_{k \geqslant n}\left|1 / \lambda_{k}-1 / \gamma_{k}\right|\right)}{\ln \max _{k \leqslant n}\left|\lambda_{k}\right|} \leqslant-a,
$$

где $a>0$ - некоторая постоянная, но пересечение $W(\Lambda) \cap W(\Gamma)$ уже не допускает спектральньй синтез. Другими словами, в основной теореме в знаменателе дроби из правой части (1.4) нельзя заменить величину $\max _{k \leqslant n}\left|\lambda_{k}\right|$ на ее логарифм, если постоянная $a$ в (6.1) мала. В частности, только сходимости ряда (2.5) уже недостаточно для справедливости основной теоремы.

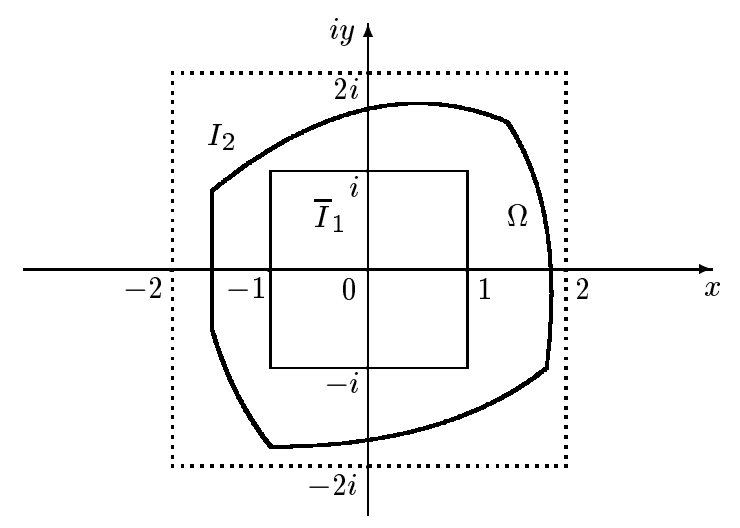

Рис. 1. К построению примера

Пусть $\Omega$ - вьпуклая область (см. рис. 1), содержашая замкнутый квадрат

$$
\bar{I}_{1}=[-1,1] \times[-i, i] \subset \Omega, \quad \pm 2, \pm 2 i \notin \Omega .
$$

Введем в рассмотрение две целые функции экспоненциального типа и вполне регулярного роста:

$$
L(z)=4 i \cos z \sin i z, \quad G(z)=4 i \cos i z \sin z
$$

с последовательностями нулей соответственно

$$
\operatorname{Zero}_{L}=\Lambda=\Lambda_{r} \cup \Lambda_{i}, \quad \operatorname{Zero}_{G}=\Gamma=\Gamma_{r} \cup \Gamma_{i},
$$


где

$$
\begin{array}{ll}
\Lambda_{r}=\left\{\lambda_{n}^{(r)}\right\}=\left\{\frac{\pi}{2}+\pi n\right\}, & \Lambda_{i}=\left\{\lambda_{k}^{(i)}\right\}=\{\pi k i\}, \\
\Gamma_{r}=\left\{\gamma_{n}^{(r)}\right\}=\{\pi n\}, & \Gamma_{i}=\left\{\gamma_{k}^{(i)}\right\}=\left\{\frac{\pi i}{2}+\pi k i\right\}, \\
n=0, \pm 1, \pm 2, \ldots, & k=0, \pm 1, \pm 2, \ldots
\end{array}
$$

Положим при $N \geqslant 1$

$$
R_{N}^{(r) \stackrel{\text { def }}{=}} \sum_{n \geqslant N}\left|\frac{1}{\lambda_{n}^{(r)}}-\frac{1}{\gamma_{n}^{(r)}}\right|=\frac{1}{2 \pi} \sum_{n \geqslant N} \frac{1}{|1 / 2+n||n|} .
$$

Тогда из первого столбца в $(6.5 \mathrm{~L}),(6.5 \mathrm{G})$ получаем ${ }^{3}$

$$
\ln R_{N}^{(r)} \sim \ln \sum_{n \geqslant N} \frac{1}{n^{2}} \sim-\ln N, \quad N \rightarrow \infty
$$

Аналогично из второго столбца в $(6.5 \mathrm{~L}),(6.5 \mathrm{G})$ получаем

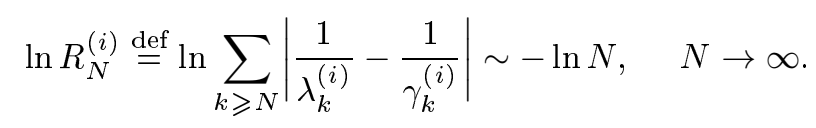

При этом

$$
\ln T_{N} \stackrel{\text { def }}{=} \ln \max \left\{\max _{n \leqslant N}\left|\lambda_{n}^{(r)}\right|, \max _{k \leqslant N}\left|\lambda_{k}^{(i)}\right|\right\} \sim \ln N, \quad N \rightarrow \infty
$$

Отсюда и из (6.6), (6.7) следует соотношение

$$
\lim _{N \rightarrow \infty} \frac{\ln \left(R_{N}^{(r)}+R_{N}^{(i)}\right)}{T_{N}}=-1 .
$$

Последнее означает, что последовательности $\Lambda$ и Г из (6.4) можно перенумеровать так, что выполнено соотношение (6.1) с постоянной $a=1$.

По закону сложения индикаторных (сопряженных) диаграмм функций вполне регулярного роста сопряженные диаграммы функций $L$ и $G$ совпадают с квадратом $\bar{I}_{1}$, определенным в $(6.2)$, т.е. содержатся в $\Omega$. Следовательно, $L$ и $G$ - характеристические функции двух функционалов $\mathscr{L}_{\text {и }} \mathscr{G}_{\text {из }} H^{*}(\Omega)$ соответственно, которые согласно представлению функций $\sin$ и $\cos$ через функцию ехр и по определению характеристических функций (0.3) действуют при свертке (0.2) по правилу

$$
\begin{aligned}
(\mathscr{L} * f)(h) & \equiv f(-1+i+h)+f(-1-i+h)-f(1+i+h)-f(1-i+h), \quad(6.8 \mathrm{~L}) \\
(\mathscr{G} * f)(h) & \equiv f(-1+i+h)+f(1+i+h)-f(-1-i+h)-f(1-i+h), \quad(6.8 \mathrm{G})
\end{aligned}
$$

\footnotetext{
${ }^{3}$ Как обычно, $A_{N} \sim B_{N}, N \rightarrow \infty$, означает, что $\lim _{N \rightarrow \infty} A_{N} / B_{N}=1$.
} 
когда $h$ пробегает геометрическую разность $\Omega \odot \bar{I}_{1}$ области $\Omega$ и квадрата $\bar{I}_{1}$ из (6.2). Инвариантное пространство решений

$$
W=W_{\mathscr{L}} \cap W_{\mathscr{G}}=W(\Lambda) \cap W(\Gamma)
$$

системы двух однородных уравнений свертки (1.5), составленной из сверток (6.8L), (6.8G), согласно (6.4), (6.5) имеет спектр $\operatorname{Spec} W=\Lambda \cap \Gamma=\{0\}$, т.е. инвариантное подпространство содержит единственную экспоненту $e^{0 \cdot z} \equiv 1$. Очевидно, $W(\{0\})$ совпадает с подпространством всех постоянных функций. Покажем, что подпространство $W$ содержит и непостоянные функции. Это и будет означать, что $W \neq W(\{0\})$, т.е. пересечение (6.9) допускающих спектральный синтез инвариантных подпространств не допускает спектральньй синтез, что и планировалось.

Рассматривая сумму и разность сверток $(6.8 \mathrm{~L}),(6.8 \mathrm{G})$, приходим к выводу, что система (1.5) эквивалентна системе уравнений свертки (в данном случае разностных уравнений)

$$
\left\{\begin{array}{rl}
f(-1+i+h)-f(1-i+h) & \equiv 0, \\
f(-1-i+h)-f(1+i+h) & \equiv 0,
\end{array} \quad h \in \Omega \ominus \bar{I}_{1},\right.
$$

т.е. $W$ совпадает с пространством решений системы (6.10). Любая двоякопериодическая мероморфная функция с двумя периодами $2 \pm 2 i$, голоморфная в открытом квадрате $I_{2}=(-2,2) \times(-2 i, 2 i)$, содержащем согласно второму условию в $(6.2)$ область $\Omega$, удовлетворяет системе (6.10), т.е. принадлежит $W$. Но среди таких функций есть и непостоянные (см. [21; гл. VII, §4]).

ЗАмЕчАНИЕ. Построенный пример подсказывает возможное направление усиления основной теоремы. Выскажем гипотезу, что в основной теореме условие (1.4) можно заменить на условие вида (6.1), где постоянная $a>0$ определенным образом связана с геометрическими характеристиками областей $D$ и $\Omega$ из основной теоремы. В [22] автором был анонсирован несколько более слабый результат, а именно: если $\Lambda$ - последовательность неединственности для выпуклой области $\Omega$, то при условии

$$
\liminf _{n \rightarrow \infty} \frac{\ln \sum_{k \geqslant n}\left|1-\lambda_{k} / \gamma_{k}\right|}{\ln \max _{k \leqslant n}\left|\lambda_{k}\right|}=-\infty
$$

пересечение $W(\Lambda) \cap W(\Gamma)$ допускает спектральный синтез. Но позже выяснилось, что первоначальное доказательство этого утверждения, к сожалению, было ошибочным. К счастью, оно нигде не публиковалось. Таким образом, высказанная вьше гипотеза пока не подтвердилась и в такой более слабой форме.

\section{Список литературы}

1. Красичков-Терновский И. Ф. Инвариантные подпространства аналитических функций. І. Спектральный синтез на вьпуклых областях // Матем. сб. 1972. Т. 87 (129). № 4. C. $459-489$.

2. Красичков-Терновский И. Ф. Инвариантные подпространства аналитических функций. II. Спектральньй синтез на вьпуклых областях // Матем. сб. 1972. Т. 88(130). №1. C. $3-30$.

3. Леонтьев A. Ф. О суммировании ряда Дирихле с комплексньми показателями и его применении // Труды МИАН. 1971. Т. 112. С. 300-326. 
4. Леонтьев $A . \Phi$. Последовательности полиномов из экспонент. М.: Наука, 1980.

5. Красичков-Терновский И. Ф. Инвариантные подпространства аналитических функций. III. О распространении спектрального синтеза // Матем. сб. 1972. Т. 88(130). №3. C. 331-352.

6. Абузярова Н. Ф. Об одном свойстве подпространств, допускающих спектральньй синтез // Матем. сб. 1999. Т. 190. № 4. С. 3-22.

7. Абузярова Н. Ф. Конечно порожденные подмодули в модулях целых функций, определяемых ограничениями на индикатор // Дис. ... канд. физ.-матем. наук. Уфа: Ин-т математики УНЦ РАН, 2000.

8. Хабибуллин Б. Н. Замкнутые идеалы и подмодули голоморфынх функций с двумя порождающими // Геометрическая теория функций и краевые задачи. Труды Математического центра им. Н.И. Лобачевского. Т. 13. Казань: Казанское матем. об-во, 2002. C. $158-163$.

9. Хабибуллин Б. Н. Замкнутые подмодули голоморфных функций, порожденные подмодулями, допускающими локальное описание // Геометрическая теория функций и краевые задачи. Труды Математического центра им. Н. И. Лобачевского. Т. 14. Казань: Казанское матем. об-во, 2002. С. 280-298.

10. Хабибуллин Б. Н. Замкнутые подмодули голоморфишт функций с двумя порождающими // Функц. анализ и его прилож. 2004. Т. 38. № 1. С. 65-80.

11. Хабибуллин Б. Н. Аппроксимационная теорема для целых функций экспоненциального типа и устойчивость нуль-последовательностей // Матем. сб. 2004. Т. 195. № 1. C. $143-156$.

12. Левин Б. Я. Распределение корней целых функций. М.: Физматгиз, 1956.

13. Levin B. Ya. Lectures on entire functions. Providence, RI: Amer. Math. Soc., 1996. (Transl. Math. Monogr. V. 150.)

14. Хабибуллин Б. Н. О росте целых функций экспоненциального типа вдоль мнимой оси // Матем. сб. 1989. Т. 180. № 5. С. 706-719.

15. Хабибуллин Б. Н. Множества единственности в пространствах целых функций одной переменной // Изв. АН СССР. 1991. Т. 55. № 5. С. 1101-1123.

16. Khabibullin B. N. Completeness of sets of complex exponentials in convex sets // Tpyды Международной конференции "Комплексньй анализ, дифференциальные уравнения и смежные вопросы”. III. Анализ и дифференциальные уравнения. Уфа: Ин-т математики с ВЦ РАН, 2000. С. 56-63.

17. Абузярова $H . \Phi$. Конечно порожденные подмодули в модуле целых функций, определяемом ограничениями на индикатор // Матем. заметки. 2002. Т. 71. № 4. С. 3-17.

18. Мандельбройт C. Ряды Дирихле. Принципш и методы. М.: Мир, 1973.

19. Сантало Л. Интегральная геометрия и геометрические вероятности. М.: Наука, 1983.

20. Титчмари Е. Теория функций. М.: Наука, 1980.

21. Лаврентьев М. Л., Шабат Б. В. Методы теории функций комплексного переменного. М.: Наука, 1987.

22. Khabibullin B.N. Spectral synthesis for the intersection of invariant subspaces of holomorphic functions // International conference on complex analysis and potential theory: Abstracts. Kiev: Institute of Mathematics of the National Academy of Ukraine, 2001. P. 24-25.

Башкирский государственный университет,

Институт математики с ВЦ УНЦ РАН, Уфа

Поступила в редакцию

E-mail: khabib-bulat@mail.ru 17.06 .2003 и 23.12 .2004 